\title{
Hyaluronidase and other Extracellular Matrix Degrading Enzymes for Cancer Therapy: New Uses and Nano-Formulations
}

Pablo Scodeller ${ }^{*}$

Sanford-Burnham Medical Research Institute, San Diego, USA

*Corresponding author: Pablo Scodeller, Sanford-Burnham Medical Research Institute. 10901 North Torrey Pines Rd. La Jolla, CA 92037 , USA, Tel: 858 646 3100; Email: pscodeller@sanfordburnham.org

Received date: Apr 04, 2014, Accepted date: Apr 26, 2014, Published date: May 7, 2014

Copyright: ( 2014 Scodeller P. This is an open-access article distributed under the terms of the Creative Commons Attribution License, which permits unrestricted use, distribution, and reproduction in any medium, provided the original author and source are credited.

\begin{abstract}
Overexpression of extracellular matrix components in tumor stroma like Hyaluronan and collagen pose a hurdle for cancer therapies. Many tumors overexpress Hyaluronic acid (HA), and Collagen. The overexpression of these components results in the elevation of a parameter called interstitial fluid pressure, which represents the resistance to the flow of a liquid. High molecular weight HA and Collagen bind water very tightly, and thus "wetting" these tumors with the drug-containing solution is impeded because it demands the displacement of those tightly bound water molecules solvating these polymers. When using conventional chemotherapy to treat a tumor, the penetration of the drug to the tissue is impoverished due to this phenomenon.
\end{abstract}

This review will collect new experimental approaches for degrading the extracellular matrix with enzymes, and the use of this as an adjuvant for conventional cancer drugs. Adjuvant systems composed of free enzyme in solution or immobilized enzyme on the surface of nanoparticles or expressed by a viral vector will be dealt with.

Keywords: Hyaluronidase; Nano-formulation; Chemoadjuvant; Collagenase; Layer-by-layer self-assembly; Extracellular matrix; Interstitial fluid pressure

\section{Abbreviations: \\ HA: Hyaluronic acid; Hyal: Hyaluronidase; ECM: Extracellular matrix; b-HABP: biotynilated Hyaluronic Acid Binding protein; IFP: Interstitial Fluid Pressure IFP; PDA: Pancreatic Ductal Carcinoma; BSA: Bovine Serum Albumin; FE-SEM: Field Emission-Scanning Electron Microscopy; MME: Macrophage Metalloelastase}

\section{Hyaluronic Acid}

Hyaluronic acid ("Hyaluronic acid", "Hyaluronan" or "HA" will be used indistinctively throughout this text) is a large, negatively charged, unbranched polymer consisting of repeating units of acetyl glucosamine and D-glucuronic acids. HA is a normal constituent in the human body, composing the extracellular matrix, and a major constituent of the joints, ovum wall, etc. In normal physiological conditions, Hyaluronan can be composed of up to 2,000-25,000 disaccharides (units), with relative molecular masses of 10e6-10e7 and polymer lengths of $2-25 \mu \mathrm{m}$ [1]. When dealing with $\mathrm{HA}$, a reference to its molecular weight must be made, since different sizes have different chemical and biological properties [2]. Turnover of HA in the body is very fast, with a half-life of less than 2 days in the skin $[3,4]$. In tissues, high $\mathrm{Mw} \mathrm{HA}$ (larger than $2 \mathrm{KDa}$ [5]) is typically detected by immunohistochemistry using biotynilated Hyaluronic Acid Binding protein (b-HABP).

High molecular weight HA solutions in water, show, by fast field cycling NMR relaxometry, three different hydration layers surrounding hyaluronan, irrespective of HA concentration. The first hydration layer was recognized to consist of strongly restrained water molecules with the slowest motion. These molecules interact with a second hydration layer which in turn is surrounded by a third layer and it is suggested that at low concentrations of $\mathrm{HA}$ in water, the amido group is involved mainly in intra-molecular water bridges, whereas at high concentrations inter-molecular water bridges become much more important justifying the non-ideal behaviour and the unusual rheological properties of the highly concentrated aqueous solutions of Hyaluronan [6].

Bauer et al. showed the antifouling properties of HA against different proteins, by measuring ellipsometric thicknesses of surfaces modified with HA, after their incubation with different proteins, as compared to the same surface without Hyaluronan [7]. The group A streptococcus surrounds itself with a capsule of Hyaluronic acid, a known virulence factor for this bacteria [8,9]. Recent reports demonstrate that HA overproduction favors the shedding of plasma membrane derived microvesicles which are suggested to play a role in tissue morphogenesis [10].

These pieces of evidence may help in understanding why HA is beneficial to tumor development and support the idea of Hyaluronic acid as an antifouling agent or "water shield".

\section{Hyaluronic Acid Overexpression in Tumors}

The overexpression of HA results in a gel-like extracellular matrix which resists the penetration of external fluid and drugs dissolved therein, and elevates the interstitial fluid pressure (IFP), as measured using the wick-in-needle method $[11,12]$ or as described by Hingorani et al. [13]

The overexpression of HA in certain tumors is backed by robust experimental evidence. Jacobetz et al. [14] have thoroughly characterized HA overexpression in tumors of different mice models by defining three categories: $+1,+2$, and +3 , according to the HA 
overexpression compared to the respective noncancerous tissue, using b-HABP immunohistochemistry on tissue sections.

A fluorescent group like Alexa Fluor 594 or ${ }^{\circ} 647$ can be coupled to Hyaluronic acid binding complex, for HA visualization using fluorescence microscopy [10].

Alcian Blue, a dye that binds acid mucopolysaccharides, is also used for Hyaluronic acid staining [15]. This procedure has evidenced Hyaluronan overexpression in different melanoma variants [16-18]. The overexpression of Hyaluronan is particularly dramatic in pancreatic tumors $[13,14]$ specially in PDA (pancreatic ductal carcinoma) one of the cancers with worse prognosis.

Hyaluronan overexpression is suggested in spontaneous canine tumors of osteosarcoma, adenoma, mastocitoma, fibrosarcoma, and neuroendocrine hepatic carcinomas [19].

In mice inoculated with tumors derived from human cell line MKN45P (gastric), HA overexpression is even higher in the rim of the tumor, as determined from Alcian Blue staining [20]. In patients with breast and ovarian carcinomas, high levels of Hyaluronan in the stroma are associated with low survival rates and elevated levels of stromal Hyaluronan are also associated with malignancy in patients who have non-small-cell lung adenocarcinomas and prostate cancer $[1,21]$. There is evidence for the beneficial role that Hyaluronan has on tumors metastatic potential [18] and the synthesis of HA was shown to be increased in mouse mammary carcinoma cell variants that exhibited high metastatic potential [22].

\section{Hyaluronidase}

Hyaluronan is cleaved by enzymes known as Hyaluronidases ("Hyaluronidase" and "Hyal" will be used indistinctively throughout this text). In humans, there are six Hyaluronidase genes, which encode Hyaluronidases that have different properties and cellular locations [1]. In mammals, Hyaluronidase is present in the head of the sperm, to degrade the ovum wall [23]. Hyal used in approved medical applications in humans is derived from bovine testis and is homologous to the one found in human sperm, PH20. Hyaluronidase is a glycosilated protein and removal of the $\mathrm{N}$-linked glycans with $\mathrm{N}$ glycosidase results in complete inactivation of enzymatic activity [24]. Isoelectric focusing of this enzyme reveals six isoforms with isoelectric points ranging between 5.1 and 6 [24]. Each oligosaccharide derived by enzymatic treatment of HA contains a double bond at the nonreducing end uronate and this functionality represents an advantage as it facilitates the detection of these HA oligosaccharides based on its absorbance in the UV at $230 \mathrm{~nm}$ [25]. Enzymatic digestion of Hyaluronic acid has been characterized by NMR, Raman, IR, and UV-Vis spectroscopy [26]. In vitro, Hyaluronidase (purified from stonefish venom) showed to degrade up to the tetra- hexa-, octa- and deca-saccharides as major end products, but not disaccharides, as characterized by HPLC and ${ }^{1}$ HNMR [27].

Enzymatic activity of Hyal is quantified using a turbidimetric assay. Under the conditions of this assay high molecular weight HA forms cloudy precipitates when incubated with Bovine Serum Albumin (BSA), while HA fragments produced by the enzymatic cleavage do not.

At low ionic strength, the catalytic activity of Hyal is complex and depends on the relative concentrations of HA, Hyal and Bovine Serum Albumin (BSA). At low ionic strength, when the ratio of Hyal to HA is low, HA binds to non-catalytic sites of Hyal [28], inactivating it. Under that situation, BSA, which is known to form a complex with HA, can restore Hyal activity.

Hyaluronidase can be specifically inhibited using apigenin [29].

Subcutaneous use of Hyal, derived from crude extracts of ovine or bovine testicular tissue, was approved by the Food and drug administration (FDA) in 1948 for treatments like hypodermoclysis [30] and as an adjunct in subcutaneous urography and treatment of vitreous hemorrhage [31]. However, this formulation is a crude extract in which less than $1 \%$ in weight is Hyaluronidase. To tackle this problem, as well as the caveat of immunogenicity in humans when using a Bovine derived extract, a recombinant human Hyal (rHuPH20) named HYLENEX ${ }^{\bullet}$ recombinant human Hyaluronidase, was introduced in the market by Halozyme [32]. Hylenex is produced by genetically engineered Chinese Hamster Ovary (CHO) cells containing a DNA plasmid encoding for a soluble fragment of human Hyaluronidase (PH20) and its use was approved by FDA in 2005 [33].

\section{Free Hyaluronidase for Cancer Therapy}

Hyaluronidases are used in cancer treatments as spreading factors for cytotoxic chemotherapy [34], they act by degrading HA, lowering the IFP and thus enhancing drug penetration [35].

It has been demonstrated by several groups that the administration of Hyal to cancerous tissues lowers the IFP within the tumors [36,38] in a non-linear concentration-dependent manner [39]. Pretreatment with bovine testis derived Hyal enhances the penetration and activity of the oncolytic drug Adriamycin in breast cancer models, in vitro and in vivo [40], and produces selective Melphalan enrichment in malignant melanomas implanted in nude mice [41]. Hyaluronidase has also shown to augment the tumor penetration of nanosized structures like liposomal Doxorubicin [42] and an oncolytic adenovirus ICOVIR5 [43], improving the efficacy of the treatments in both cases. The use of Hyal as an additive in therapy comprises not only chemotherapy but extends also to radioimmunotherapy applications: Hyaluronidase has been used as adjuvant for the delivery of 125I-labeled TP-3 monoclonal antibody against an osteosarcomaassociated antigen [37].

In vitro, Hyal also favors the cytotoxicity of anticancer drugs [44].

Phase II clinical trials are under way to treat patients with Pancreatic Ductal Carcinoma (a type of tumor with high overexpression of Hyaluronic acid [13]) with a combination of Pegylated recombinant human $\mathrm{rHuPH} 20$, known as PEGPH20, and the drug Gemcitabine, intravenously injected. The latest results of this trial are encouraging [45].

Using genetic engineering, a modified version of oncolytic human adenovirus, ICOVIR5, expressing PH20 Hyaluronidase, denoted as ICOVIR 17, enhanced the spread of the virus within the tumor respect to the non Hyal-containing virus and concomitantly enhanced suppression of tumor growth [43]. In a more recent study by the same group [19], a new, RGD motif-containing version of ICOVIR17 was constructed (the tripeptide RGD binds to av integrins, which are overexpressed in tumor entothelia [45]). This new system proved very effective in treating different spontaneous tumors in dogs [19].

\section{Immobilized Hyaluronidase for Cancer Therapy}

Hyaluronidase can be presented to the target tissue in a multivalent display by using nanotechnology to synthesize nanosized water 
dispersible nanostructures which are later derivatized with the enzyme. The use of nanotechnology provides a controllable and versatile approach to construct different systems at will, of different sizes and shapes and provides control over the way the enzyme is presented on the surface. Through "wet", bottom-up synthesis, one can produce different shaped nanostructures, like elongated ones, which are known to enhance the affinity for the target compared to the spherical shape [46,47]. Hyaluronidase may be attached to the surface of the nanostructure by chemical coupling or using the layer-by-layer self-assembly technique.

Multivalent interactions of Hyal with the target tissue and a lower diffusion coefficient of the nanosized object are expected to provide a longer residence time of Hyal in contact with the substrate and result in a more effective degradation of HA.

To demonstrate that the multivalent presentation of Hyal is indeed more effective than using the free enzyme, Scodeller et al. have described the first nanoformulation of Hyaluronidase [17]. In that work, melanoma tumors were pretreated with solutions of equal enzymatic activities of free and immobilized Hyal, and the authors showed that the immobilized Hyaluronidase was more effective than the free enzyme for their treatment study. The degrading properties of these particles showed to be quite dramatic when analyzing the tissues under Field Emission-Scanning Electron Microscopy (FE-SEM) even after only one application of the Hyal-modified nanoparticles (Figure $1 \mathrm{~A}$ and $\mathrm{B})$

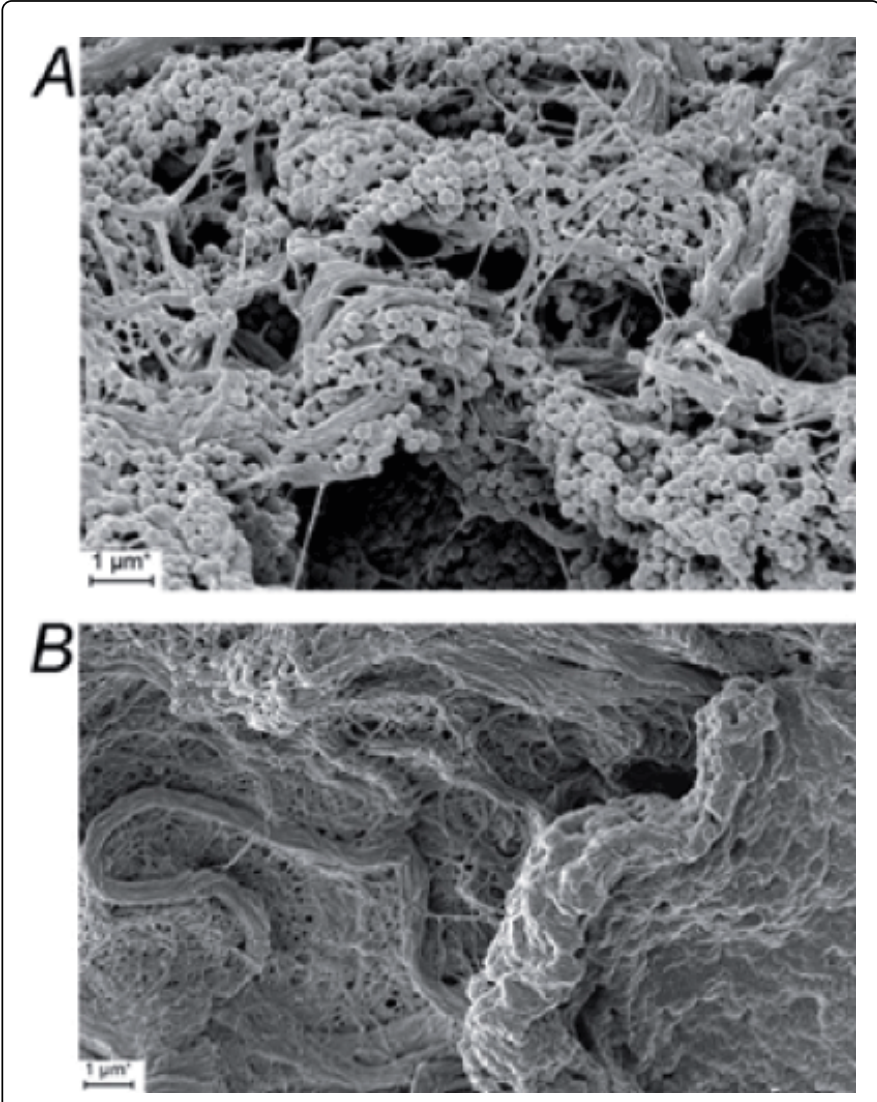

Figure 1: Surface of A375 tumors as seen with FE-SEM, 21 days after injection of cells. (A) Two hours after one injection of Hyalmodified particles. (B) Two hours after one injection of PBS.
In that work, human melanoma A375 inoculated tumors were treated with Carboplatin, with the free and immobilized Hyal pretreaments using peritumoral injections. To functionalize these particles with Hyal the authors used the layer-by-layer (LBL) selfassembly technique [48] which consists of the alternate adsorption of polycations and polyanions from water solutions, where each layer deposition is accompanied by charge reversal as a result of charge overcompensation [49]. The LBL technique was first used by Caruso et al. to successfully functionalize micrometer sized colloidal surfaces with enzymes, preserving enzymatic activity [50], and the group of Calvo was the first to successfully functionalize nanometer colloidal gold surfaces with enzymes, conserving catalytic activity [51].

This LBL technique is the most appealing when functionalizing surfaces with enzymes since enzymatic activity is maintained and multiple layers may be deposited to yield a larger response; this method can be employed to construct catalytically active enzymatic multilayers by self-assembling enzymes and polymers on planar [52] and colloidal surfaces [51]. The catalytic activity of this multilayer will depend on enzymatic loading, enzyme orientation and mobility, parameters which are directly controlled by the deposition conditions (assembly pH and ionic strength) [53]. Also, importantly, many times the specific enzymatic activity (enzymatic activity/moles of enzyme) is increased upon surface immobilization. Monteiro et al. [54] have immobilized Hyal on Langmuir Blodgett films prepared with the sodium salt of dihexadecylphosphoric acid, ending with dipalmitoylphosphatidylcholine. In that study, tests of enzymatic activity, using Hyaluronic acid as the substrate, showed an increase of activity compared to the homogeneous medium and the authors propose a model of protein insertion with Hyal-lipid chains hydrophobic interactions and the hydrophilic site containing the active site, exposed to the solution.

The particles used by Scodeller et al. are $250 \mathrm{~nm}$ sized silica spheres, of low cost and ease to synthesize and which have proved to be biocompatible [55]. Currently, a fluorescent version of this same system is being studied, in which silica nanoparticles are replaced with a fluorescent Fluorescein core protected by a shell of silica matrix [56] which provides a very photostable signal and allows for long term live imaging of these particles [57].

In this nanoformulated Hyal, a smaller diffusion coefficient and multivalent interactions (affinity scales exponentially with number of valences) with the target tissue is the proposed mechanism to explain the enhanced adjuvant effect respect to free Hyal.

\section{Other Extracellular Matrix Degrading Enzymes}

Other ECM components, namely, Collagen I and Laminin I are overexpressed in certain types of cancers like breast tumors, as evidenced by immunohistochemistry [58,59]. In breast cancer the overexpression of these ECM components is higher at the rim of the solid tumor and is accompanied by mechanical stiffening as measured with nano-indentation [58].

Collagenase has been used to favor the penetration of DNA in tumors [60]. In this work by Cemazar et al. the authors test Collagenase, Hyaluronidase, and the combination of both, to increase the transfection efficiency of gene electrotransfer in different cancer models, in vitro and in-vivo. The combination of enzymes provided higher effeciency than any one of them alone, for the transfection of: LPB fibrosarcoma, SA-1 fibrosacroma and EAT carcinoma tumors. Whereas the use of Hyal alone was more efficient for transfection in 
B16 melanoma tumors, where only traces of Collagen are present. In an LPB tumor treatment study, the combination of enzymes also resulted in the highest volume reduction.

Macrophage metalloelastase (MME) has been used as adjuvant for intra-tumoral injection of oncolytic adenovirus [61]. In this study, the administration of oncolytic adenovirus combined with MME significantly improved the treatment results in a subcutaneous mouse model of colorectal carcinoma in comparison with single treatments with either agent alone.

\section{Conclusion and Outlook}

Extracellular matrix degrading enzymes greatly enhance the dispersion of drugs within many tumors and improve the outcome of the treatments. Additional improvement in the treatment has been accomplished by using a nano-formulation of the enzyme.

The choice of the ECM degrading enzyme to be used as adjuvant will depend on the type of tumor, according to the ECM component it overexpresses. In the case of Collagenase, this should be specifically targeted to the tumor, to avoid Collagen degradation in healthy tissue which has a half-life of approximately fifteen years [62].

Nanotechnology will be necessary to take these enzyme-aided therapies to a new level: It will allow combining the enzyme, the drug and adding imaging capability in the same system. For example, by functionalizing the surfaces of, drug-carrying, mesoporous silica nanoparticles [63] or responsive polymersome [64], with the enzyme, or a combination of enzymes. It will allow to specifically targeting the enzyme to the tissue of interest by incorporating tumor homing peptides [65] or antibodies in the nanosystem. It will enhance the interaction with the target substrate through the multivalency, possibly prolonging the enzyme's residence time in the region of interest. All of these features could translate into a more efficient cancer therapy.

\section{Acknowledgements}

Acknowledgements to E.A. Scodeller and M.A. Bonder for careful proofreading of the manuscript and to P. Saudade for his words.

\section{References}

1. Toole BP (2004) Hyaluronan: from extracellular glue to pericellular cue. Nat Rev Cancer 4: 528-539.

2. Stern R, Asari AA, Sugahara KN (2006) Hyaluronan fragments: an information-rich system. Eur J Cell Biol 85: 699-715.

3. Laurent UB, Dahl LB, Reed RK (1991) Catabolism of hyaluronan in rabbit skin takes place locally, in lymph nodes and liver. Exp Physiol 76: 695-703.

4. Fraser JR, Laurent TC (1989) Turnover and metabolism of hyaluronan. Ciba Found Symp 143: 41-53.

5. http://www.emdmillipore.com/life-science-research/hyaluronic-acid-binding-protein-bovinenasal-cartilage-biotinylated/EMD_BIO-85911/p_BAOb.s1LVlwAAAEWMWIfVhTm

6. PrÅ ${ }^{-} \operatorname{sov} \tilde{A}_{i} A$, Conte $P$, Kucer $\tilde{A}^{k}$, Alonzo G (2010) Dynamics of hyaluronan aqueous solutions as assessed by fast field cycling NMR relaxometry. Anal Bioanal Chem 397: 3023-3028.

7. Bauer S, Arpa-Sancet MP, Finlay JA, Callow ME, Callow JA, et al. (2013) Adhesion of marine fouling organisms on hydrophilic and amphiphilic polysaccharides. Langmuir 29: 4039-4047.

8. Bisno AL, Brito MO, Collins CM (2003) Molecular basis of group A streptococcal virulence. Lancet Infect Dis 3: 191-200.

9. Wessels MR, Moses AE, Goldberg JB, DiCesare TJ (1991) Hyaluronic acid capsule is a virulence factor for mucoid group A streptococci. Proc Natl Acad Sci U S A 88: 8317-8321.

10. Rilla K, Pasonen-Seppänen S, Deen AJ, Koistinen VV, Wojciechowski S, et al. (2013) Hyaluronan production enhances shedding of plasma membrane-derived microvesicles. Exp Cell Res 319: 2006-2018.
11. Fadnes HO, Reed RK, Aukland K (1977) Interstitial fluid pressure in rats measured with a modified wick technique. Microvasc Res 14: 27-36.

12. Heldin CH, Rubin K, Pietras K, Ostman A (2004) High interstitial fluid pressure - an obstacle in cancer therapy. Nat Rev Cancer 4: 806-813.

13. Provenzano PP, Cuevas C, Chang AE, Goel VK, Von Hoff DD, et al. (2012) Enzymatic targeting of the stroma ablates physical barriers to treatment of pancreatic ductal adenocarcinoma. Cancer Cell 21: 418-429.

14. Jacobetz MA, Chan DS, Neesse A, Bapiro TE, Cook N, et al. (2013) Hyaluronan impairs vascular function and drug delivery in a mouse model of pancreatic cancer. Gut 62: 112-120.

15. Sisca RF, Langkamp HH, Thonard JC (1971) Histochemical demonstration of acid mucopolysaccharide production by tissue cultured epithelial-like cells. Histochemie 27: 173-181.

16. Turley EA, Tretiak M (1985) Glycosaminoglycan production by murine melanoma variants in vivo and in vitro. Cancer Res 45: 5098-5105.

17. Scodeller P, Catalano PN, Salguero N, Duran H, Wolosiuk A, et al. (2013) Hyaluronan degrading silica nanoparticles for skin cancer therapy. Nanoscale 5: 9690-9698.

18. Zhang L, Underhill CB, Chen L (1995) Hyaluronan on the surface of tumor cells is correlated with metastatic behavior. Cancer Res 55: 428-433.

19. Laborda E, Puig-Saus C2, Rodriguez-García A2, Moreno R2, Cascalló M3, et al. (2014) A pRbresponsive, RGD-modified, and hyaluronidase-armed canine oncolytic adenovirus for application in veterinary oncology. Mol Ther 22: 986-998.

20. Scodeller, P. Unpublished data

21. Toole BP (2009) Hyaluronan-CD44 Interactions in Cancer: Paradoxes and Possibilities. Clin Cancer Res 15: 7462-7468.

22. Kimata K, Honma Y, Okayama M, Oguri K, Hozumi M, et al. (1983) Increased synthesis of hyaluronic acid by mouse mammary carcinoma cell variants with high metastatic potential. Cancer Res 43: 1347-1354.

23. Swyer GI (1947) The hyaluronidase content of semen. Biochem J 41: 409-413.

24. Li MW, Yudin AI, Robertson KR, Cherr GN, Overstreet JW (2002) Importance of glycosylation and disulfide bonds in hyaluronidase activity of macaque sperm surface PH-20. J Androl 23: 211-219.

25. Sakai S, Hirano K, Toyoda H, Linhardt RJ, Toida T (2007) Matrix assisted laser desorption ionization-time of flight mass spectrometry analysis of hyaluronan oligosaccharides. Anal Chim Acta 593: 207-213.

26. Alkrad JA, Mrestani Y, Stroehl D, Wartewig S, Neubert R (2003) Characterization of enzymatically digested hyaluronic acid using NMR, Raman, IR, and UV-Vis spectroscopies. J Pharm Biomed Anal 31: 545-550.

27. Sugahara K, Yamada S, Sugiura M, Takeda K, Yuen R, et al. (1992) Identification of the reaction products of the purified hyaluronidase from stonefish (Synanceja horrida) venom. Biochem J 283 : 99-104.

28. Lenormand H, Tranchepain F, Deschrevel B, Vincent JC (2009) The hyaluronan-protein complexes at low ionic strength: how the hyaluronidase activity is controlled by the bovine serum albumin. Matrix Biol 28: 365-372.

29. Liu D, Pearlman E, Diaconu E, Guo K, Mori H, et al. (1996) Expression of hyaluronidase by tumor cells induces angiogenesis in vivo. Proc Natl Acad Sci U S A 93: 7832-7837.

30. Dunn AL, Heavner JE, Racz G, Day M (2010) Hyaluronidase: a review of approved formulations, indications and off-label use in chronic pain management. Expert Opin Biol Ther 10: 127-131.

31. Hyaluronidase Monograph (Amphadase, Hydase, Vitrase, Hylenex) Final, in: National PBM Drug Monograph, VHA PharmacyBenefits Management Service and the Medical Advisory Panel, Washington, DC, 2008 .

32. www.halozyme.com

33. http://www.drugs.com/history/hylenex.html

34. Baumgartner G, Gomar-Hass C, Sakr L, Ulsperger E, Wogritsch C (1998) The impact of extracellular matrix on the chemoresistance of solid tumors-experimental and clinical results of hyaluronidase as additive to cytostatic chemotherapy. Cancer Lett. 131(1): 85-99.

35. Stern R (2008) Hyaluronidases in cancer biology. Semin Cancer Biol 18: 275-280.

36. Heldin CH, Rubin K, Pietras K, Ostman A (2004) High interstitial fluid pressure - an obstacle in cancer therapy. Nat Rev Cancer 4: 806-813.

37. Brekken C, Hjelstuen MH, Bruland S, de Lange Davies C (2000) Hyaluronidase-induced periodic modulation of the interstitial fluid pressure increases selective antibody uptake in human osteosarcoma xenografts. Anticancer Res. 20(5B): 3513-3519

38. Whatcott CJ, Han H, Posner RG, Hostetter G, Von Hoff DD (2011) Targeting the tumo microenvironment in cancer: why hyaluronidase deserves a second look. Cancer Discov 1: 291-296. 
Citation: Scodeller (2014) Hyaluronidase and other Extracellular Matrix Degrading Enzymes for Cancer Therapy: New Uses and NanoFormulations . J Carcinog Mutage 5: 178. doi:10.4172/2157-2518.1000178

Page 5 of 5

39. Brekken C, de Lange Davies C (1998) Hyaluronidase reduces the interstitial fluid pressure in solid tumours in a non-linear concentration-dependent manner. Cancer Lett 131: 65-70.

40. Beckenlehner K, Bannke S, Spruss T, Bernhardt G, Schönenberg H, et al. (1992) Hyaluronidase enhances the activity of adriamycin in breast cancer models in vitro and in vivo. J Cancer Res Clin Oncol 118: 591-596.

41. Muckenschnabel I, Bernhardt G, Spruss T, Buschauer A (1996) Hyaluronidase pretreatment produces selective melphalan enrichment in malignant melanoma implanted in nude mice. Cancer Chemother Pharmacol. 38(1):88-94.

42. Eikenes L, Tari M, Tufto I, Bruland OS, et al. (2005) Hyaluronidase induces a transcapillary pressure gradient and improves the distribution and uptake of liposomal doxorubicin (Caelyx) in human osteosarcoma xenografts. Br J Cancer. 93(1): 81-88

43. Guedan S, Rojas JJ, Gros A, Mercade E, Cascallo M, et al. (2010) Hyaluronidase expression by an oncolytic adenovirus enhances its intratumoral spread and suppresses tumor growth. Mol Ther 18: $1275-1283$.

44. St Croix B, Man S, Kerbel RS (1998) Reversal of intrinsic and acquired forms of drug resistance by hyaluronidase treatment of solid tumors. Cancer Lett 131: 35-44.

45. http://www.clinicaltrials.gov (search term: pegph20)

46. Ruoslahti E (2002) Specialization of tumor vasculature. Nat Rev Cancer. 2(2):83-90.

47. Park JH, von Maltzahn G, Zhang L, Schwartz MP, Ruoslahti E, et al. (2008) Magnetic Iron Oxide Nanoworms for Tumor Targeting and Imaging. Adv Mater. 20(9): 1630-1635.

48. Kolhar P, Anselmo AC, Gupta V, Pant K, Prabhakarpandian B, et al. (2013) Using shape effects to target antibody-coated nanoparticles to lung and brain endothelium.Proc Natl Acad Sci U S A. 110(26):10753-10758

49. G. Decher (1997) Fuzzy Nanoassemblies: Toward Layered Polymeric Multicomposites. Science 277, 1232-1237.

50. F. Caruso, H. Lichtenfeld, E. Donath and H. Mohwald (1999) Investigation of Electrostatic Interactions in Polyelectrolyte Multilayer Films: Binding of Anionic Fluorescent Probes to Layer Assembled onto Colloids. Macromolecules 32, 2317-2328.

51. Scodeller P, Flexer V, Szamocki R, Calvo EJ, Tognalli N, et al. (2008) Wired-enzyme core-shell Au nanoparticle biosensor. J Am Chem Soc 130: 12690-12697.

52. Scodeller P, Carballo R, Szamocki R, Levin L, Forchiassin F, et al. (2010) Layer-by-layer selfassembled osmium polymer-mediated laccase oxygen cathodes for biofuel cells: the role of hydrogen peroxide. J Am Chem Soc 132: 11132-11140.
53. Flexer V, Forzani ES, Calvo EJ, Ludueña SJ, Pietrasanta LI (2006) Structure and thicknes dependence of "molecular wiring" in nanostructured enzyme multilayers. Anal Chem 78: 399-407.

54. Monteiro DS, Nobre TM, Zaniquelli ME (2011) Hyaluronidase behavior at the air/liquid and air/ lipid interfaces and improved enzymatic activity by its immobilization in a biomembrane model. J Phys Chem B 115: 4801-4809.

55. Botella P, Abasolo I, Fernández Y, Muniesa C, Miranda S, et al. (2011) Surface-modified silica nanoparticles for tumor-targeted delivery of camptothecin and its biological evaluation. J Control Release 156: 246-257.

56. Gulay Durgun, Kasim Ocakoglu , and Serdar Ozcelik (2011) Systematic Tuning the Hydrodynamic Diameter of Uniformed Fluorescent Silica Nanoparticles. J. Phys. Chem. C 115 (33), 16322-16332.

57. Scodeller, P. Unpublished data

58. Plodinec M, Loparic M, Monnier CA, Obermann EC, Zanetti-Dallenbach R, et al. (2012) The nanomechanical signature of breast cancer. Nat Nanotechnol 7: 757-765.

59. Chauhan VP, Martin JD, Liu H, Lacorre DA, Jain SR, et al. (2013) Angiotensin inhibition enhances drug delivery and potentiates chemotherapy by decompressing tumour blood vessels. Nat Commun 4: 2516

60. Cemazar M, Golzio M, Sersa G, Escoffre JM, Coer A, et al. (2012) Hyaluronidase and collagenase increase the transfection efficiency of gene electrotransfer in various murine tumors. Hum Gene Ther 23: 128-137.

61. Lavilla-Alonso S, Bauer MM, Abo-Ramadan U, Ristimäki A, Halavaara J, et al. (2012) Macrophage metalloelastase (MME) as adjuvant for intra-tumoral injection of oncolytic adenovirus and its influence on metastases development. Cancer Gene Ther 19: 126-134.

62. Verzijl N, DeGroot J, Thorpe SR, Bank RA, Shaw JN, et al. (2000) Effect of collagen turnover on the accumulation of advanced glycation end products. J Biol Chem 275: 39027-39031.

63. Meng H, Liong M, Xia T, Li Z, Ji Z, et al. (2010) Engineered design of mesoporous silica nanoparticles to deliver doxorubicin and P-glycoprotein siRNA to overcome drug resistance in a cancer cell line. ACS Nano 4: 4539-4550.

64. Pegoraro C, Cecchin D, Gracia LS, Warren N, Madsen J, et al. (2013) Enhanced drug delivery to melanoma cells using PMPC-PDPA polymersomes. Cancer Lett 334: 328-337.

65. Ruoslahti E (2012) Peptides as targeting elements and tissue penetration devices for nanoparticles. Adv Mater 24: $3747-375^{6}$. 